
\title{
Research S Surare \\ The Study of Ocular Parameters in Low to Moderate Myopic Chinese Children Age Between Eight to Eighteen-Year-Old
}

\section{Zhengxuan Li}

Beijing Institute of Ophthalmology, Capital Medical University

Lu Sun

Beijing Institute of Ophthalmology, Capital Medical University

Hongxin Song ( $\square$ songhongxin2012@ccmu.edu.cn )

Beijing Institute of Ophthalmology, Capital Medical University

\section{Research Article}

Keywords: Myopia, Corneal parameters, Overnight orthokeratology lens

Posted Date: July 28th, 2021

DOI: https://doi.org/10.21203/rs.3.rs-736271/v1

License: (c) (i) This work is licensed under a Creative Commons Attribution 4.0 International License.

Read Full License 


\section{Abstract}

Purpose: The primary aim of this study was to analyze the distribution of ocular parameters of children aged from eight to eighteen-year-old with low to moderate myopia as well as offer useful information for the design and clinical fitting of ortho-k lens in China.

Methods: In this retrospective study, we collected data of 300 subjects (600 eyes) age between eight to eighteen-year-old from the myopic control outpatient clinic at Beijing Tongren Hospital, Beijing, China. The spherical equivalent (SE), axial length (AL), central corneal thickness (CCT), anterior chamber depth (ACD), corneal endothelial cell density (CECD) \corneal diameter (CD), simulated K (Sim K) were collected from all the subjects and analyzed.

Results: The mean of SE for all subjects was $-3.12 \pm 1.21 \mathrm{D}$, the median was $-3.16 \mathrm{D}$. The mean of AL for all subjects was $24.72 \pm 0.83 \mathrm{~mm}$, the median was $24.72 \mathrm{~mm}$. The mean of CCT for all subjects was $537.8 \pm 29.82 \mu \mathrm{m}$, the median was $538.17 \mu \mathrm{m}$. The mean of CECD for all subjects was $3340.3 \pm 352.6$ cells $/ \mathrm{mm}^{2}$, the median was 3357.77 cells $/ \mathrm{mm}^{2}$. The mean of ACD for all subjects was $3.71 \pm 0.21 \mathrm{~mm}$, the median was $3.69 \mathrm{~mm}$. The mean of $\mathrm{CD}$ for all subjects was $12.15 \pm 1.67 \mathrm{~mm}$, the median was 12.05 $\mathrm{mm}$. The mean of flat $\mathrm{K}$ was $42.80 \pm 1.29$, the median was 42.81 . The mean of steep $\mathrm{K}$ was $43.76 \pm 1.37$, the median was 43.76 .

Conclusions: This study provided the distribution of ocular parameters to offer useful information for diagnosis of many eye conditions. Meanwhile, the distribution of ocular parameters of children aged from eight to eighteen-year-old with low to moderate myopia is beneficial to make the application of overnight orthokeratology lens more appropriate and effective.

\section{Background}

Together with the sclera, the cornea forms the outermost layer. Cornea, as one of the most essential links, must ensure its transparency, so there is no blood vessel in the cornea tissue. [1, 2] Blood vessels end at the limbus of the cornea to form a vascular network, and nutrients expand into the cornea from of the eyeball-fibrous tunic. Cornea with aqueous humor, lens, vitreous body constitutes the indispensable way for light to enter the inner eyes. The five layers of the cornea are: epithelium, lamina elastica anterior, stroma, lamina elastica posterior and endothelium.[3-5] The ocular parameters are of great significance for diagnosis of keratoconus, glaucoma, diabetes and other systemic diseases manifests in eyes. [6]

Myopia is the most universal ocular refractive disorder all over the world, especially in China. It was reported that myopia prevalence was $80-90 \%$ in young adults, and $10-20 \%$ young adults developed into high myopia.[7-9] National prevalence of myopia increased from primary to high school continued the same trend.[10-13] Overnight orthokeratology (ortho-k) lens has been regarded as a safe and effective method to control the myopia progression. Therefore, understanding the distribution of ocular parameters in low to moderate myopic Chinese adolescents is important in ortho-k lens design and fitting. Ocular parameters has been studied in adults in different population, such as in Caucasians, Nigerians and 
Chinese.[14-16] Chan and colleagues studied the corneal parameters in Chinese children aged between six and twelve years old in Hongkong where is southern part of China in 2012, and this study mainly focused on the corneal anterior parameters, also this study was performed almost ten years ago, many ocular parameters in population may have changed. [17] To provide more detailed ocular information, we collected the ocular parameters of children aged from eight to eighteen-year-old with low to moderate myopia from Beijing Tongren Hospital myopic control clinic, Beijing, China, and we analyzed the ocular parameters distribution in these subjects. The distribution of ocular parameters will provide useful information for the design and application of the ortho-k lens in China.

\section{Methods}

Subjects' data were collected from the children who came to the myopic control clinic at Beijing Tongren Hospital between January 2018 and July 2019. The inclusion criteria were children age between eight to eighteen-year-old, with no history of contact lens worn, and free of history of intraocular surgery or ocular trauma, with myopia no more than - 6.00 D and astigmatism (negative cylinder) lower than - 3.50 D. Best corrected vision was $20 / 20$ or better. The study adheres to the Declaration of Helsinki and was approved by Beijing Tongren Hospital Review Board and Ethics Committee.

IOL-Master (Carl Zeiss, Germany) was used in axial length measurement and corneal diameter, corneal topography (Medmont E300 topography, Medmont Pty Ltd, Vermont, VIC, Australia) was used to collect the cornea topography data. Corneal endothelium density was measured by a noncontact specular microscope Topcon Robo SP-9000 (Konan, Hyogo, Japan). Statistical analysis was performed using SPSS 19.0 (SPSS, Inc, Chicago, IL, USA). All data were presented in the shape of the mean \pm Std Dev. All distributions were shown in the form of a box graph.

\section{Results}

Three hundred subjects' data were collected in our study. Figure 1 shows the distribution of spherical equivalent (SE), axial length (AL), central corneal thickness (CCT), corneal endothelial cell density (CECD), anterior chamber depth (ACD), corneal diameter (CD) and simulated K (Sim K).

The mean of SE for all subjects was $-3.12 \pm 1.21 \mathrm{D}$, the median was $-3.16 \mathrm{D}$. The mean of AL for all subjects was $24.72 \pm 0.83 \mathrm{~mm}$, the median was $24.72 \mathrm{~mm}$. The mean of CCT for all subjects was $537.8 \pm$ $29.82 \mu \mathrm{m}$, the median was $538.17 \mu \mathrm{m}$. The mean of CECD for all subjects was $3340.3 \pm 352.6 \mathrm{cells} / \mathrm{mm}^{2}$, the median was 3357.77 cells $/ \mathrm{mm}^{2}$. The mean of ACD for all subjects was $3.71 \pm 0.21 \mathrm{~mm}$, the median was $3.69 \mathrm{~mm}$. The mean of CD for all subjects was $12.15 \pm 1.67 \mathrm{~mm}$, the median was $12.05 \mathrm{~mm}$. The mean of flat $K$ for was $42.80 \pm 1.29$, the median was 42.81 . The mean of steep $K$ for was $43.76 \pm 1.37$, the median was 43.76. (Table 1) In the comparison of different genders and ages (Table 2\&3), no statistical significance has been found $(\mathrm{P}>0.05)$. Table 1. The results of the ocular parameters of the subjects (see Additional table 1) 
Table 2. The distribution of the ocular parameters in male and female subjects (see Additional table 2)

Table 3. The distribution of the above ocular parameters by decade of age (see Additional table 3)

$S E=$ spherical equivalent,$A L=$ axial length,$C C T=$ central corneal thickness, $C E C D=$ corneal endothelial cell density, $A C D=$ anterior chamber depth, $C D=$ corneal diameter, $\operatorname{Sim} K=$ simulated $K$.

Figure 1 The distribution of spherical equivalent $(A)$, axial length(B), central corneal thickness $(C)$, corneal endothelial cell density $(D)$, corneal diameter $(E)$, Flat $K(F)$, Steep $K(G)$ and anterior chamber depth $(H)$ in Chinese juveniles (see Additional Fig. 1)

\section{Discussion}

\section{To make the application of overnight orthokeratology lens in China become more appropriate and effective}

With the increase of the pace of life and the pressure of the learning, the myopia rate of Chinese children has been presented a sharp rise in the recent years. Reducing and controlling the myopia progression has become a hot topic among researchers all over the world. [18-20] Overnight orthokeratology lens is an effective method in slowing down the progression of myopia. As an external object flatten the central portion of the cornea, the fitting of overnight orthokeratology lens needs the precise data of the ocular parameters of patients, especially for the juveniles who are in the period of growth and development. [21-26]

The main application market of contact lenses was for conveniently refractive correction and cosmetic reason. Therefore, the target customers of contact lens are designed for adults, the research and application of ocular parameters are also based on their data.[17] However, with the prevalence of myopia development, especially the high incidence rate of myopia in East Asia, Overnight orthokeratology lens has gradually become an effective means to control the development of myopia. $[9,27,28]$

In this study, statistical analysis was conducted in groups of different genders (males, 126 subjects with 252 eyes; females, 174 subjects with 348 eyes), simultaneously in age divided younger or equal to ten years old (180 subjects with 360 eyes) and older than ten years old (120 subjects with 240 eyes). No statistical significance was found in the above conditions.

Overnight orthokeratology lens, as a contact lens which were placed against the surface of the cornea, are worn at night and take off during the daytime. The distribution of these ocular parameters is benefit for the first best fitting of overnight orthokeratology lens. The axial elongation is an important factor for evaluating the progression of myopia, while the elongation of the AL does not mean the severity of myopia because there is also a physiological increase in adolescents.[29-32] Studying the distribution of $\mathrm{AL}$ in adolescents is helpful to understand the physiological axial growth. 
The fitting of ortho-k lens requires accurate measurements of ocular parameters to achieve a proper lens fitting, the variation of ocular parameters was crucial for the practitioner to select the appropriate ortho-k lens. Most of the lenses now offered by manufacturers are based on the corneal profile of Caucasian adults, and repeated adjustments and lens fitting due to the improper lens would cost tremendous money and time. $[9,27,30]$ Our study provides the distribution of ocular parameters in low to moderate myopic Chinese adolescents; therefore, it can provide useful information for contact lens design for Asian children.

\section{To compare with previous studies of different race.}

Although the distribution of the corneal parameters of Asians, Caucasians, Africans and Arabian have well manifested in the range of adults in the past 20 years (table 4), there was still a shortage of large sample aimed at children. The retrospective study found that Asians have smaller corneal diameter than Caucasians and Africans. The corneal diameter in our study is larger than previous study indicating there may be ocular parameters changes during the past decade. Previous studies demonstrate that Chinese children have thinner central corneal thickness than others, no conspicuous difference with the results of our study. The data of characteristics of the corneal endothelial cell density was similar with previous studies.

Table 4. The comparison of ocular parameters with previous studies of different race (see Additional table 4)

$N R=$ Not reported

\section{Conclusion}

In conclusion, our study provides the distribution of ocular parameters in low to moderate myopic Chinese adolescents to offer useful information for the design and clinical fitting of ortho-k lens in China.

\section{Abbreviations}

SE: spherical equivalent; AL: axial length(B); CCT: central corneal thickness; CECD: corneal endothelial cell density(D); CD: corneal diameter(E); ACD: anterior chamber depth(H); Sim K: simulated K

\section{Declarations}

\section{Ethics approval and consent to participate}

The study adheres to the Declaration of Helsinki and was approved by Beijing Tongren Hospital Review Board and Ethics Committee.

\section{Availability of data and materials}


The datasets during and/or analyzed during the current study are available from the corresponding author on reasonable request.

Competing Interest

No conflicting relationship exists for any author.

\section{Funding}

No funding

\section{Authors' contribution}

$\mathrm{Li}$, Sun and Song were primarily responsible for experimental concept and design. Li and Sun performed data acquisition and analysis, as well as drafting of the manuscript. Li was also involved in data analysis. All authors reviewed and approved the final manuscript.

\section{Consent for publication}

Not applicable.

\section{References}

1. Meek KM, Knupp C: Corneal structure and transparency. Prog Retin Eye Res 2015, 49:1-16.

2. Doughty MJ, Jonuscheit S: Corneal structure, transparency, thickness and optical density (densitometry), especially as relevant to contact lens wear-a review. Cont Lens Anterior Eye 2019, 42(3):238-245.

3. Eghrari AO, Riazuddin SA, Gottsch JD: Overview of the Cornea: Structure, Function, and Development. Prog Mol Biol Transl Sci 2015, 134:7-23.

4. DelMonte DW, Kim T: Anatomy and physiology of the cornea. J Cataract Refract Surg 2011, 37(3):588-598.

5. Zhang B, Xue Q, Li J, Ma L, Yao Y, Ye H, Cui Z, Yang H: 3D bioprinting for artificial cornea: Challenges and perspectives. Med Eng Phys 2019, 71:68-78.

6. Ma J, Wang Y, Wei P, Jhanji V: Biomechanics and structure of the cornea: implications and association with corneal disorders. Surv Ophthalmol 2018, 63(6):851-861.

7. Tay SA, Farzavandi S, Tan D: Interventions to Reduce Myopia Progression in Children. Strabismus 2017, 25(1):23-32.

8. Morgan IG, French AN, Ashby RS, Guo X, Ding X, He M, Rose KA: The epidemics of myopia: Aetiology and prevention. Prog Retin Eye Res 2018, 62:134-149.

9. Cho P, Tan Q: Myopia and orthokeratology for myopia control. Clin Exp Optom 2019, 102(4):364-377. 
10. Liu HH, Xu L, Wang YX, Wang S, You QS, Jonas JB: Prevalence and progression of myopic retinopathy in Chinese adults: the Beijing Eye Study. Ophthalmology 2010, 117(9):1763-1768.

11. He M, Zeng J, Liu Y, Xu J, Pokharel GP, Ellwein LB: Refractive error and visual impairment in urban children in southern china. Invest Ophthalmol Vis Sci 2004, 45(3):793-799.

12. Zhang M, Li L, Chen L, Lee J, Wu J, Yang A, Chen C, Xu D, Lam DS, Sharma A et al: Population density and refractive error among Chinese children. Invest Ophthalmol Vis Sci 2010, 51(10):4969-4976.

13. David Troilo KTaEH: Imposed Anisometropia, Accommodation, and Regulation of Refractive State. 2009, 86:31-39.

14. Mashige KP: A review of corneal diameter, curvature and thickness values and influencing factors*. African Vision and Eye Health 2013, 72(4).

15. Sanchis-Gimeno JA, Sanchez-Zuriaga D, Martinez-Soriano F: White-to-white corneal diameter, pupil diameter, central corneal thickness and thinnest corneal thickness values of emmetropic subjects. Surg Radiol Anat 2012, 34(2):167-170.

16. Hassan Hashemi MK, Kamran Yazdani, Shiva Mehravaran, Kazem Mohammad, and Akbar Fotouhi,: White-to-White Corneal Diameter in the Tehran Eye Study. clinical science 2010, 29:9-12.

17. Chan KY, Cheung SW, Cho P: Corneal parameters of six-to 12-year-old Chinese children. Clin Exp Optom 2012, 95(2):160-165.

18. Chen C, Cheung SW, Cho P: Myopia control using toric orthokeratology (TO-SEE study). Invest Ophthalmol Vis Sci 2013, 54(10):6510-6517.

19. Chen Q, Li M, Yuan Y, Me R, Yu Y, Shi G, Ke B: Interaction between Corneal and Internal Ocular Aberrations Induced by Orthokeratology and Its Influential Factors. Biomed Res Int 2017, 2017:3703854.

20. Chen R, Mao X, Jiang J, Shen M, Lian Y, Zhang B, Lu F: The relationship between corneal biomechanics and anterior segment parameters in the early stage of orthokeratology: A pilot study. Medicine (Baltimore) 2017, 96(19):e6907.

21. Swarbrick HA: Orthokeratology review and update. Clin Exp Optom 2006, 89(3):124-143.

22. Cho P, Cheung SW, Edwards M: The longitudinal orthokeratology research in children (LORIC) in Hong Kong: a pilot study on refractive changes and myopic control. Curr Eye Res 2005, 30(1):71-80.

23. Walline JJ, Jones LA, Sinnott LT: Corneal reshaping and myopia progression. Br J Ophthalmol 2009, 93(9):1181-1185.

24. Kakita T, Hiraoka T, Oshika T: Influence of overnight orthokeratology on axial elongation in childhood myopia. Invest Ophthalmo/ Vis Sci 2011, 52(5):2170-2174.

25. Jacinto Santodomingo-Rubido CV-C, Bernard Gilmartin and Ramón Gutiérrez-Ortega: Myopia Control with Orthokeratology Contact Lenses in Spain (MCOS): Refractive and Biometric Changes. The Association for Research in Vision and Ophthalmology 2012.

26. Cho P, Cheung SW: Retardation of myopia in Orthokeratology (ROMIO) study: a 2-year randomized clinical trial. Invest Ophthalmol Vis Sci 2012, 53(11):7077-7085. 
27. He M, Du Y, Liu Q, Ren C, Liu J, Wang Q, Li L, Yu J: Effects of orthokeratology on the progression of low to moderate myopia in Chinese children. BMC Ophthalmol 2016, 16:126.

28. Cheung SW, Boost MV, Cho P: Pre-treatment observation of axial elongation for evidence-based selection of children in Hong Kong for myopia control. Cont Lens Anterior Eye 2019, 42(4):392-398.

29. Chen Z, Zhou J, Qu X, Zhou X, Xue F: Effects of orthokeratology on axial length growth in myopic anisometropes. Cont Lens Anterior Eye 2018, 41(3):263-266.

30. Lipson MJ, Brooks MM, Koffler BH: The Role of Orthokeratology in Myopia Control: A Review. Eye Contact Lens 2018, 44(4):224-230.

31. Kinoshita N, Konno Y, Hamada N, Kanda Y, Shimmura-Tomita M, Kakehashi A: Additive effects of orthokeratology and atropine $\mathbf{0 . 0 1 \%}$ ophthalmic solution in slowing axial elongation in children with myopia: first year results. Jpn J Ophthalmo/2018, 62(5):544-553.

32. Zhang Y, Chen Y: Effect of Orthokeratology on Axial Length Elongation in Anisomyopic Children. Optom Vis Sci 2019, 96(1):43-47.

\section{Figures}



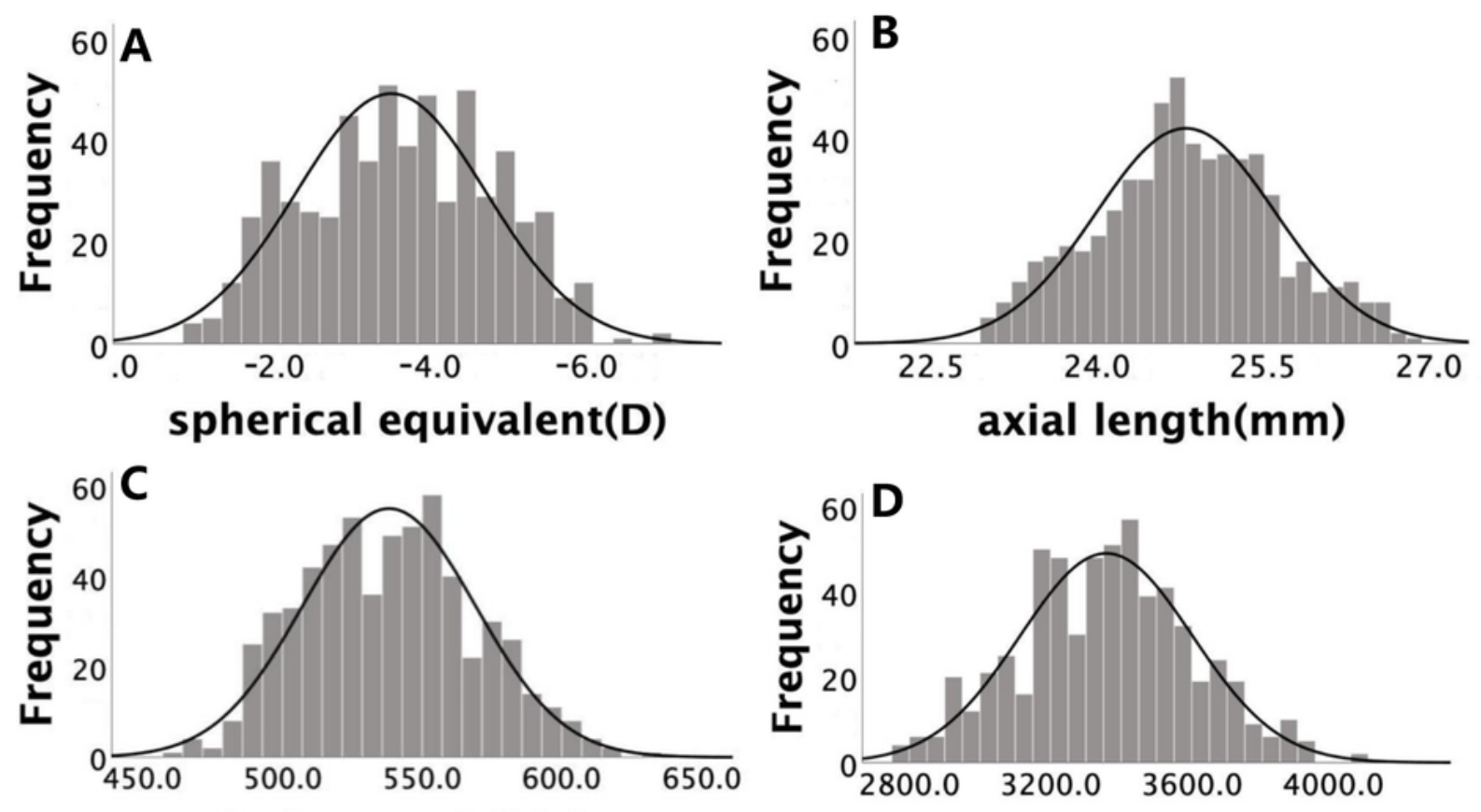

central corneal thickness

$(\mu \mathrm{m})$
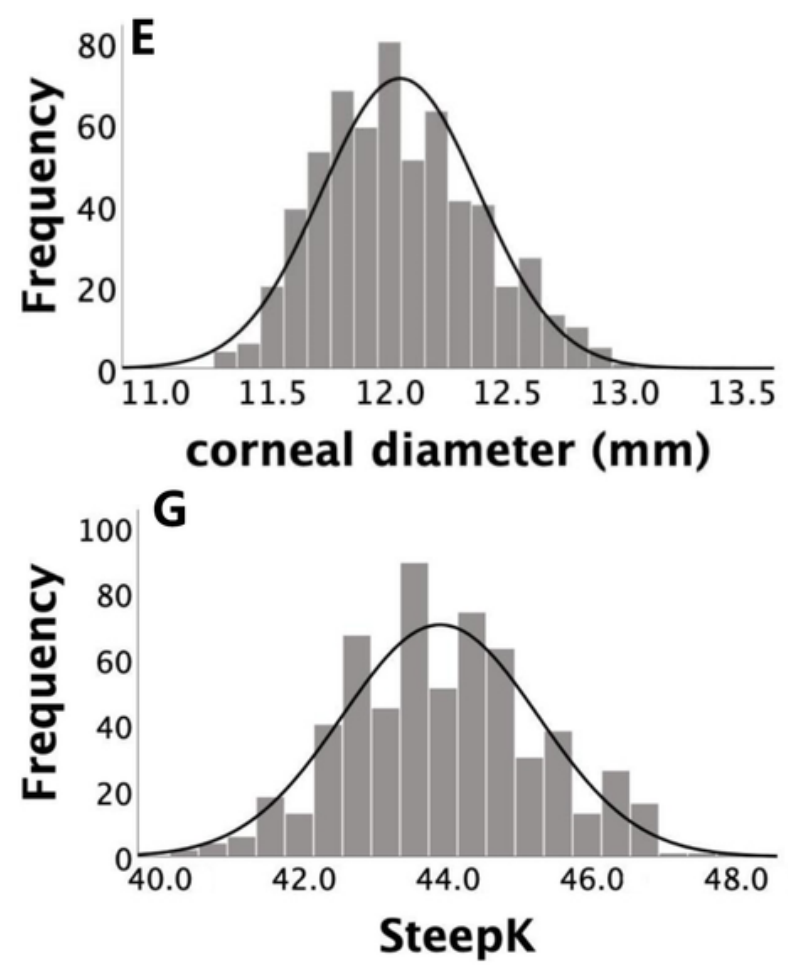

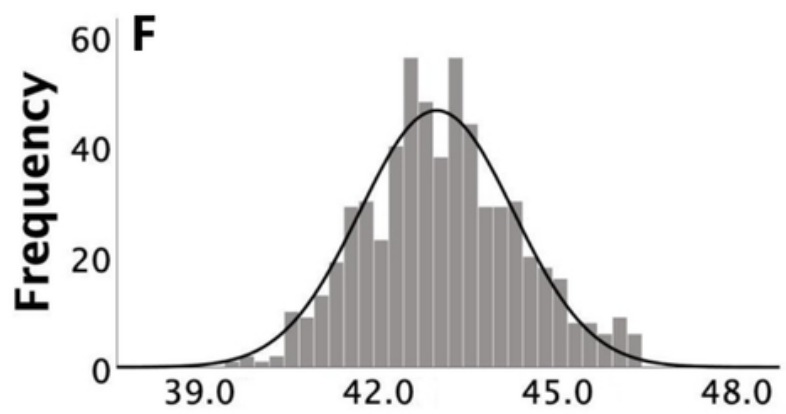

FlatK

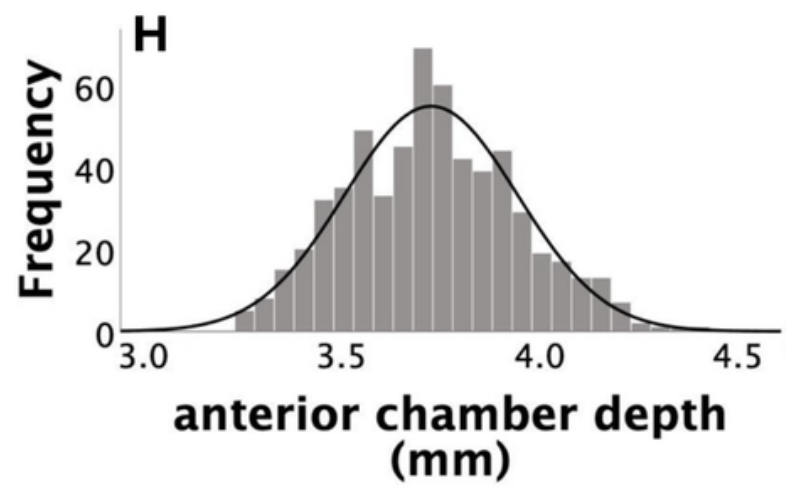

Figure 1

The distribution of spherical equivalent(A), axial length(B), central corneal thickness(C), corneal endothelial cell density $(D)$, corneal diameter $(E)$, Flat $K(F)$, Steep $K(G)$ and anterior chamber depth $(H)$ in Chinese juveniles 
This is a list of supplementary files associated with this preprint. Click to download.

- additionaltable1.xIsx

- additionaltable2.xlsx

- additionaltable3.xlsx

- additionaltable4.xlsx 